\title{
Partial bileaflet resection and reconstruction of mitral valve in acute infective endocarditis
}

\author{
Igor E. Konstantinov, MD, PhD, Perth, Australia
}

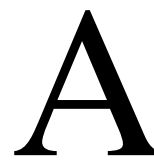

cute infective endocarditis of the mitral valve (MV) in noncompliant intravenous drug users presents a difficult problem. Although progressive sepsis despite appropriate antibiotic therapy necessitates prompt sur-

From the Department of Cardiothoracic Surgery, Sir Charles Gairdner Hospital, Perth, Australia.

Received for publication Dec 23, 2007; accepted for publication Jan 13, 2008 .

Address for reprints: Igor E. Konstantinov, MD, PhD, Department of Cardiothoracic Surgery, Sir Charles Gairdner Hospital, G Block, Hospital Ave, Perth, WA 6009 Australia.

J Thorac Cardiovasc Surg 2008;136:533-4

$0022-5223 / \$ 34.00$

Copyright (C) 2008 by The American Association for Thoracic Surgery doi:10.1016/j.jtcvs.2008.01.029 gical intervention, the risk of prosthetic endocarditis after valve replacement is very high in this group of noncompliant patients. Excessive tissue damage and annular abscess, on the other hand, may prevent simple conventional repair. I refer to the procedure as 'reconstruction' as opposed to 'repair', when $1 / 3$ or more of the valve has to be removed. Reconstruction of the MV, as described here, could be a valuable alternative under these circumstances.

\section{Clinical Summary}

A 28-year-old man with a long history of intravenous drug use was admitted with fever and chills. Multiple blood cultures demonstrated Staphylococcus aureus. Echocardiography demonstrated a large, mobile vegetation straddling both leaflets of the MV and an abscess extending into the posterior MV annulus (Figure 1, $A$ and $B$ ). Prolapse of the posterior MV leaflet as a result of rupture of the infected chordae
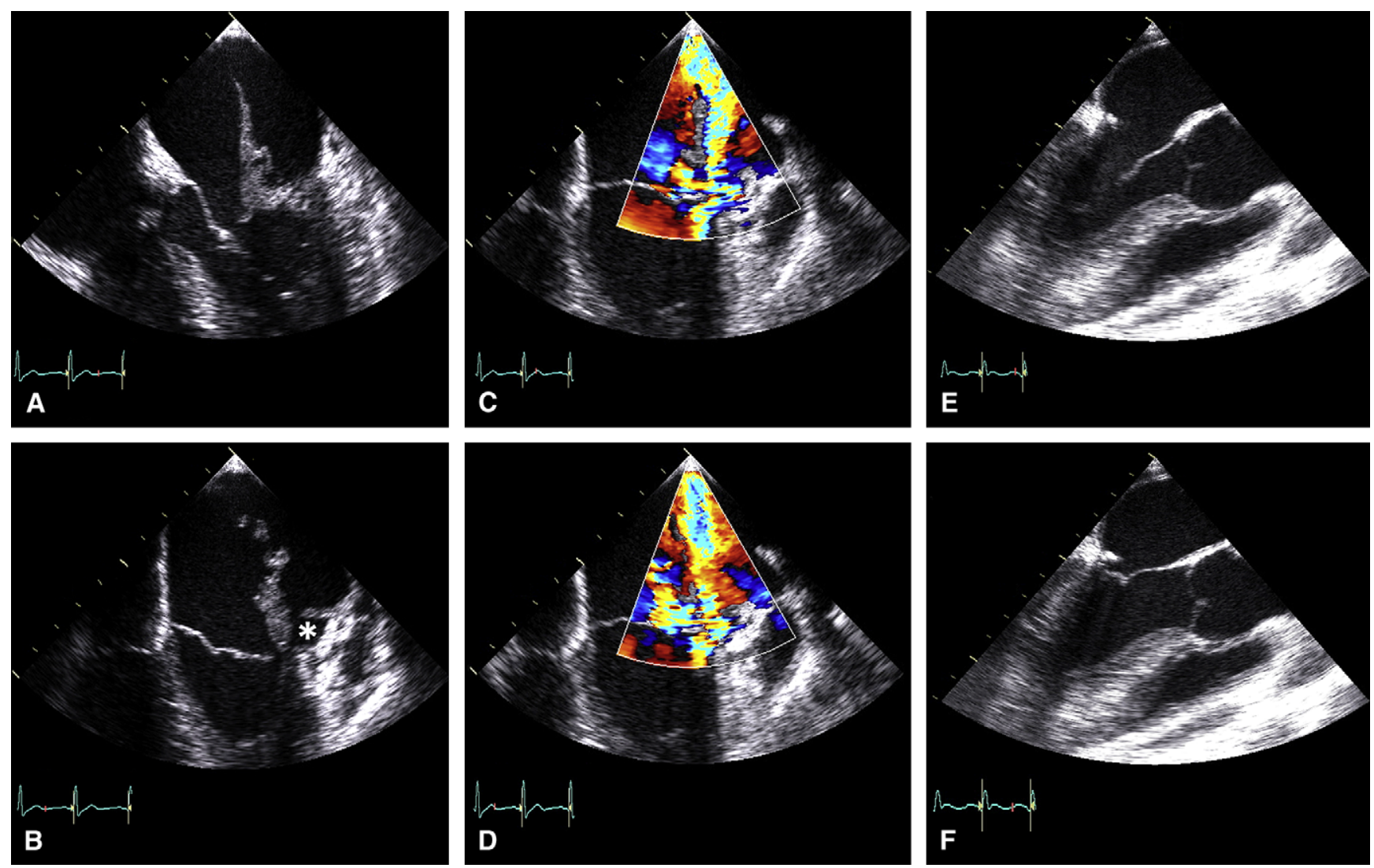

Figure 1. Transesophageal echocardiograms demonstrating large vegetation straddling both leaflets of mitral valve (A) and abscess cavity (asterisk) of posterior portion of mitral annulus (B), paravalvular leak through ruptured abscess (C), severe mitral regurgitation combining transvalvular and paravalvular jets (D), and postreconstruction anatomy ( $E$ and $F)$. 


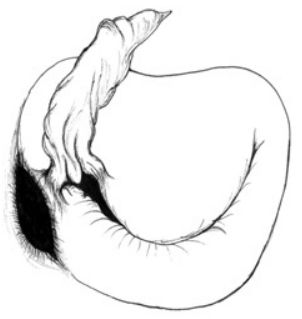

A

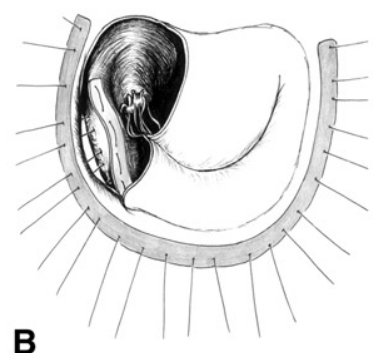

B

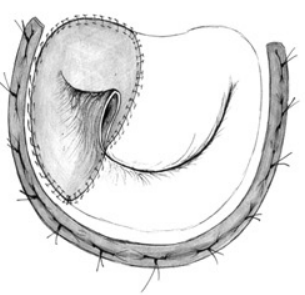

C

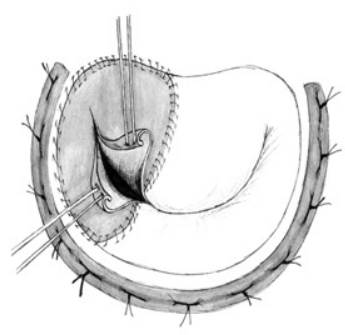

D

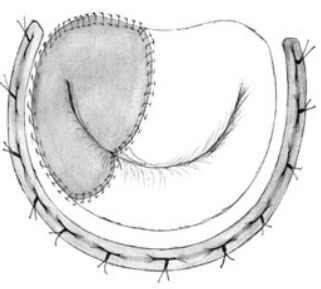

$\mathbf{E}$

Figure 2. Schematic illustrating technique of mitral valve reconstruction. A, Large vegetation and area of ruptured abscess of mitral annulus are seen. B, Complete resections of $A 1$ and P1 segments of mitral valve are performed, and abscess cavity is obliterated between two strips of autologous pericardium. C, Defect is patched with autologous pericardium with excessive hood. D, Hood of pericardium is incised, and polytetrafluoroethylene chordae are placed at free edges of pericardial patch. $E$, Mitral valve reconstruction is completed by chordal implantation and annuloplasty with strip of pericardium.

resulted in severe mitral regurgitation. Despite appropriate antibiotic coverage, sepsis developed and the abscess ruptured, resulting in additional severe paravalvular regurgitation (Figure 1, $C$ and $D$ ). When acute pulmonary edema developed, the patient underwent urgent surgery.

Autologous pericardium was harvested and soaked in rifampin. A large vegetation straddling both leaflets and a drained abscess cavity immediately adjacent to the left fibrous trigone were seen (Figure 2, $A$ ). Resections of the $\mathrm{A} 1$ and $\mathrm{P} 1$ segments of the MV and the entire subvalvular apparatus of these segments were performed. The abscess was obliterated between two strips of autologous pericardium (Figure 2, B). The MV was reconstructed with autologous pericardium soaked in rifampin (Figure 2, $C-E$ ) and GORE-TEX chordae (W.L. Gore \& Associates, Inc, Flagstaff, Ariz). A strip of autologous pericardium facilitated MV annuloplasty. Apart from 4-0 GORETEX chordae and 4-0 Prolene sutures (Ethicon, Inc, Somerville, $\mathrm{NJ}$ ), no artificial materials were used. An additional vegetation was found inside the left atrial appendage, which was resected. Postoperative echocardiography demonstrated normal function of reconstructed MV (Figure 1, $E$ and $F$ ). The patient is free of symptoms at 8 months of follow-up and has only trivial mitral insufficiency.

\section{Discussion}

Prompt surgical treatment is generally preferred in cases of acute MV endocarditis in association with uncontrolled intracardiac spread and sepsis. ${ }^{1}$ Extensive involvement of both leaflets and the annulus of the MV in a noncompliant patient is a difficult problem. Replacement of the valve with prosthesis carries a high risk of prosthetic endocarditis. Recurrences of endocarditis are common. Although successful repair of acute MV endocarditis involving a single leaflet has been reported, extensive reconstruction after partial resection of both leaflets of the MV is a much more challenging procedure that may require placement of a multiple neochordae to ensure competence of the MV. A simplified technique for multiple chordal placement for the entire chordal apparatus of the MV has been described. ${ }^{2,3}$ Extensive destruction of the MV and its chordal attachments, débridement of all infected tissues, and subsequent reconstruction of both leaflets with pericardial patch necessitate placement of neochordae. The absence of any adjacent native chordae that could be used as a reference point, however, makes the use of the previously described technique of multiple chordal replacement problematic. GORE-TEX neochordae therefore need to be sized individually. The elegant technique of making individually measured artificial GORE-TEX chordae was first described for MV repair by Brizard and Carpentier. ${ }^{4}$ The details of the technique of individually premeasuring chordae have been illustrated previously. ${ }^{5}$ The technique of extensive bileaflet valvular reconstruction described here may be a valuable alternative for the noncompliant patient with acute infective endocarditis.

I am grateful to Mr Graham Jenkins and Dr Trenton Barrett for their help in preparing the illustrations.

\section{References}

1. de Kerchove L, Vanoverschelde JL, Ponclet A, Glineur D, Rubay J, Zech F, et al. Reconstructive surgery in acute mitral endocarditis: feasibility, safety and durability. Eur J Cardiothorac Surg. 2007;31:592-9.

2. Konstantinov IE, Jalali H. Total chordal augmentation in a child with Marfan syndrome and severe mitral insufficiency. Tex Heart Inst J. 2007:34:423-4.

3. Tam R, Joshi P, Konstantinov IE. A simple method of preparing artificial chordae for mitral valve repair. J Thorac Cardiovasc Surg. 2006;132: 1486-7.

4. Brizard C, Carpentier A. Congenital malformation of the mitral valve. In: Stark JF, De Leval MR, Tsang VT, eds. Surgery for congenital heart defects. 3rd ed. Chichester, UK: John Wiley \& Sons; 2006. p. 573-90.

5. Konstantinov IE. Total resection and complete reconstruction of tricuspid valve in acute infective endocarditis. J Thorac Cardiovasc Surg 2008; In press. 